\title{
Chemodynamical evolution of galaxies with hypernovae
}

\author{
Chiaki Kobayashi \\ National Astronomical Observatory of Japan, 2-21-1 Osawa, Mitaka-shi, Tokyo 181-8588, \\ Japan; email: chiaki@th.nao.ac.jp
}

\begin{abstract}
We simulate the formation and evolution of galaxies with a hydrodynamical model including supernova and hypernova feedback. The large contribution of hypernovae is required from the observed abundance ratios in the Milky Way Galaxy. The hypernova feedback suppress star formation efficiently, which results in the cosmic star formation rate history peaked at $z \sim 4$. It also drives galactic outflows efficiently in low mass galaxies, and these winds eject heavy elements into the intergalactic medium. The ejected baryon and metal fraction is larger for less massive galaxies, which results in the mass-metallicity relation of galaxies. We also simulate the chemodynamical evolution of the Milky Way Galaxy, and show the difference of the stellar populations in the bulge and disk.
\end{abstract}

Keywords. galaxies: abundances, galaxies: evolution, methods: n-body simulations

\section{Introduction}

While the evolution of the dark matter is reasonably well understood, the evolution of the baryonic component is much less certain because of the complexity of the relevant physical processes, such as star formation and feedback. With the commonly employed, schematic star formation criteria alone, the predicted star formation rates (SFRs) are higher than what is compatible with the observed luminosity density. Thus feedback mechanisms are in general invoked to reheat gas and suppress star formation. We include both supernova and hypernova feedback in our hydrodynamical model in this paper (see Kobayashi, Springel \& White 2007, hereafter K07, for the details).

Supernovae eject not only thermal energy but also heavy elements into interstellar medium. Thus the star formation history is imprinted in the chemical abundances of stars and galaxies. Different types of supernovae, i.e., Type II and Ia supernovae (SNe II and Ia), produce different heavy elements with different timescales. Recently, it is found that hypernovae $(\mathrm{HNe})$, which have more than ten times larger explosion energy $\left(E_{51} \gtrsim 10\right)$, produce a certain amount of iron.

Kobayashi et al. (2006) calculated the nucleosynthesis yields for wide ranges of metallicity and energy, based on the light curve and spectra fitting of individual supernovae. With these yields, the elemental abundance ratios are in good agreement with observations from oxygen to zinc. Especially, the observed abundance of $\mathrm{Zn}([\mathrm{Zn} / \mathrm{Fe}] \sim 0)$ can be explained only by a large contribution of HNe. We adopt these yields and energies in our hydrodynamical model.

\section{Cosmological Simulations}

We simulate the evolution of gas and stellar systems and the chemical enrichment from SNe II, SNe Ia, and HNe, from the cosmological initial condition with $H_{0}=70 \mathrm{~km}$ $\mathrm{s}^{-1} \mathrm{Mpc}^{-1}, \Omega_{m}=0.3, \Omega_{\Lambda}=0.7, \Omega_{\mathrm{b}}=0.04, n=1$, and $\sigma_{8}=0.9$. We use an SPH code GADGET-2 by Springel (2005), and introduce the metal-dependent cooling rates 

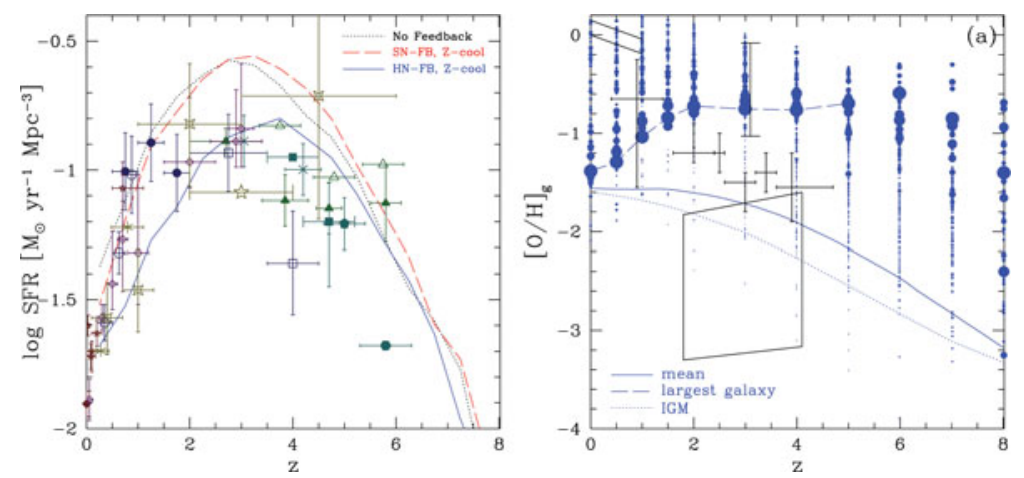

Figure 1. (a) Cosmic star formation rates for no feedback case (dotted line) and for the SN (dashed line) and HN (solid line) feedback with the metal-dependent cooling. See K07 for the references of Rest-frame U-band, IR, and $\mathrm{H} \alpha$ observations. Rest-frame UV observations are plotted with dust correction (Steidel et al. 1999). (b) Redshift evolution of iron abundances of gas. The points show individual galaxies with the size representing the size of galaxies. The solid, dashed, and dotted lines are for the mean, the largest galaxy in the simulated region, and the IGM, respectively. See the text for the observational data (errorbars, box).

(Sutherland \& Dopita 1993) and chemical enrichment scheme by Kobayashi (2004). The initial condition is calculated in a $10 h^{-1}$ Mpc cubic box with periodic boundary conditions with $N_{\mathrm{DM}}=N_{\text {gas }}=96^{3}$. We adopt the Salpeter IMF, the star formation timescale $c_{*}=0.1$, and the number of feedback neighbors $N_{\mathrm{FB}}=405$.

Figure 1a shows the cosmic SFRs that are directly measured from the ages of stellar particles for the low resolution simulations. Supernova feedback (dashed line) decreases the SFR from $z \sim 3$, but metal-dependent cooling increases as large as no feedback case (dotted line). If we include hypernova feedback (solid line), the SFR starts to be suppressed from $z \sim 6$ onwards, and is overall smaller by a factor of 3 at $0 \lesssim z \lesssim 3$. The resulting SFR is in broad agreement with the observations that show a peak of $\log \left(\mathrm{SFR} /\left[\mathrm{M}_{\odot} \mathrm{yr}^{-1}\right]\right) \sim-1$ at $z \sim 4$.

Without feedback, $25 \%$ of baryons turn into stars, which is too large. With SN feedback, the stellar fraction reduces to $10-15 \%$. The larger energy ejection by HNe results in the stellar fraction less than $10 \%$, which is consistent with the recent observational estimate (Fukugita \& Peebles 2004). The present mean gas metallicity is $[\mathrm{Fe} / \mathrm{H}] \sim-1.3$ for SN feedback, and is reduced to $\sim-1.8$ for $\mathrm{HN}$ feedback. The mean stellar metallicity is almost solar for the SN feedback, and becomes sub-solar for the HN feedback.

The metal enrichment timescale depends on the environment. Figure $1 \mathrm{~b}$ shows the evolution of oxygen abundances in the gas phase in different environments. In large galaxies, enrichment takes place so quickly that $[\mathrm{O} / \mathrm{H}]$ reaches $\sim-1$ at $z \sim 7$, which is consistent with the sub-solar metallicities of the Lyman break galaxies (large errorbar at $z=3$, Pettini et al. 2001). The low metallicities of DLA systems (errorbars, Prochaska et al. 2003) are also consistent with our galaxies, provided these systems are dwarf galaxies or the outskirts of massive galaxies. The low $[\mathrm{C} / \mathrm{H}]$ of the IGM (box, Schaye et al. 2003) can be explained if the IGM is enriched only by SNe II and HNe. The average metallicity of the universe reaches $[\mathrm{O} / \mathrm{H}] \sim-2$ and $[\mathrm{Fe} / \mathrm{H}] \sim-2.5$ at $z \sim 4$, but reaches the same values at $z \sim 3$ in the IGM.

When and where do stars form? To answer this question, we break up the cosmic SFR history according to galaxy mass (see Figs. 13 and 14 in K07). As a result, the majority of the stars in present-day massive galaxies have formed in smaller systems at high redshifts, and the stellar population of these galaxies is as old as $10 \mathrm{Gyr}$, consistent 

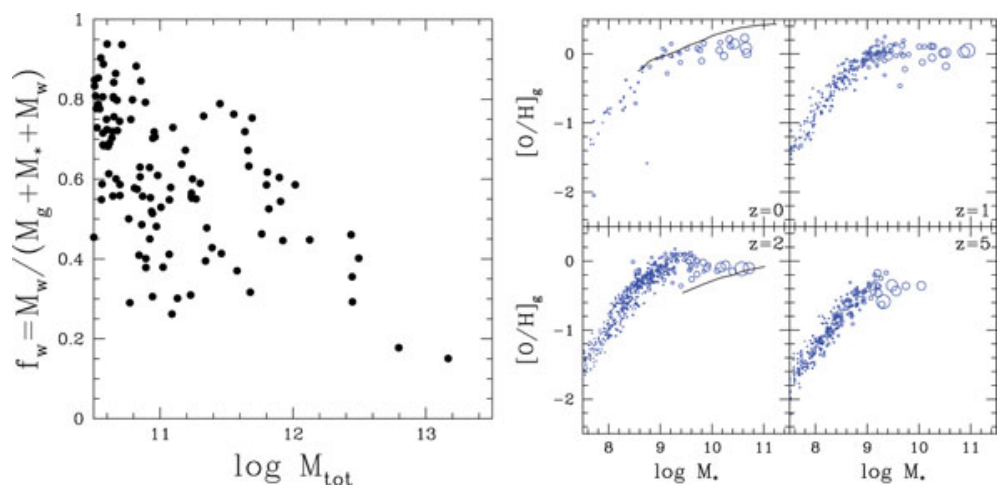

Figure 2. (a) The wind fraction $f_{\mathrm{w}}=M_{\mathrm{w}} /\left(M_{\mathrm{g}}+M_{*}+M_{\mathrm{w}}\right)$ against the total mass. (b) Mean metallicities of cold gas $\left(T<10^{4} \mathrm{~K}\right)$ within $10 \mathrm{kpc}$, plotted against the total stellar mass at $z=0,1,2$, and 5 . See the text for the observational data (solid lines).

with observations of elliptical galaxies. No relation is found between luminosity-weighted age and stellar mass. The ages of low-mass galaxies span a wide range of $1-10$ Gyr.

How are heavy elements ejected from galaxies to the IGM? In the simulation, we can trace the orbit of gas particles over time. Exploiting this, we define as wind particles those that are not in galaxies now, but have been in galaxies before (see Fig. 15 in K07). In this simulation, $\sim 10 \%$ of baryons turn into stars, $\sim 10 \%$ of the gas stays in galaxies ( $\sim 8 \%$ is hot), and $\sim 20 \%$ is ejected as galactic winds. The rest, half of the baryons, never accretes onto galaxies.

When we follow the orbits of gas particles, we can also examine from which galaxies the wind gas particles are ejected. This allows a measurement of the ejected wind mass from each galaxy. In Figure 2a, we plot the wind fraction, i.e. the ratio between the total wind mass to the total accreted baryon mass, against the total mass. A clear relation is found between the wind fraction and the total mass. Winds are efficiently ejected from small galaxies, with $\sim 80 \%$ of accreted baryons being ejected from $M_{\text {tot }} \sim 10^{11} \mathrm{M}_{\odot}$ galaxies. A similar relation is also found for the ejected metal fraction, i.e. the ratio between the wind metal mass to the total metal mass. It is interesting that the wind fraction and the ejected metal fraction correlate well with the stellar metallicity. Based on this finding, we conclude that the origin of the mass-metallicity relation can be explained with the galactic winds.

In galaxies, metallicity of the cold gas increases with galaxy mass (Fig.2b), which is comparable to observations with a large scatter (solid line at $z=0$, Tremonti et al. 2004; $z=2$, Erb et al. 2006). The central cold gas shows a relation between galaxy mass and metallicity with shallower slope than observed in emission-line galaxies. For the stellar population, the observed mass-metallicity relation is well reproduced (Kobayashi \& Arimoto 1999; Pahre et al. 1998; Gallazzi et al. 2005), and originates in mass-dependent galactic winds. These relations are present since $z \sim 5$.

\section{Chemodynamical Evolution of the Milky Way Galaxy}

We simulate the chemodynamical evolution of the Milky Way Galaxy, using the special purpose computer GRAPE for gravity and the SPH method for hydrodynamics (see Kobayashi, Nakasato, \& Nomoto 2007, in preparation). The initial condition is similar to those in Kobayashi $(2004,2005)$, but with the initial angular momentum of $\lambda \sim 0.1$, the total mass of $\sim 10^{12} M_{\odot}$, and $\sim 60000$ particles. We choose an initial condition where the galaxy does not undergo major mergers, otherwise no disk galaxy can form. 

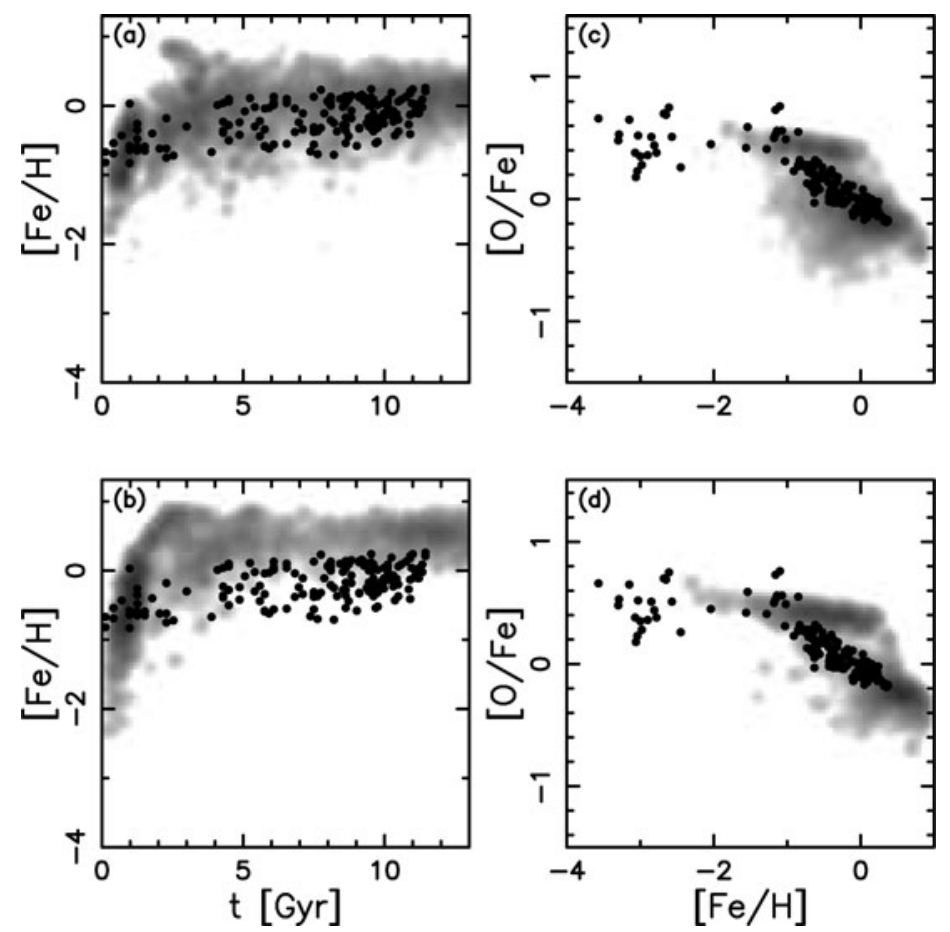

Figure 3. Age-metallicity relations ( $\mathrm{a}$ and $\mathrm{b}$ ) and $[\mathrm{O} / \mathrm{Fe}]-[\mathrm{Fe} / \mathrm{H}]$ relations $(\mathrm{c}$ and $\mathrm{d}$ ) in the solar neighborhood ( $a$ and $c$ ) and bulge (b and d). The contours show the mass density for the simulation. The dots show the observations of stars in the solar neighborhood (see the text for data sources).

A disk galaxy forms through the successive merging of subgalaxies with various masses. The merging of subgalaxies induces the initial starburst and the bulge forms by $z \gtrsim 3$. According to the late gas accretion, the majority of disk stars form at $z \lesssim 2$. The bulge has the de Vaucouleurs surface brightness profile with the effective radius of $\sim 1.5 \mathrm{kpc}$, and the disk has the exponential profile with $\sim 6 \mathrm{kpc}$.

The age-metallicity relations are shown in Figure 3ab. (a) In the solar neighborhood $(r=7.5-8.5 \mathrm{kpc},|z| \leqslant 0.5 \mathrm{kpc}),[\mathrm{Fe} / \mathrm{H}]$ increases to $\sim 0$ at $t \sim 2 \mathrm{Gyr}$, which is consistent with the observations (Edvardsson et al. 1993). (b) In the bulge ( $r \leqslant 1 \mathrm{kpc}$ ), $[\mathrm{Fe} / \mathrm{H}]$ increases more quickly.

The $[\mathrm{O} / \mathrm{Fe}]-[\mathrm{Fe} / \mathrm{H}]$ relations are shown in Figure 3cd. (c) In the solar neighborhood, we can reproduce the observational trend (Edvardsson et al. 1993, Bensby et al. 2004, Gratton et al. 2003, Cayrel et al. 2004). [O/Fe] decreases because of the delayed iron enrichment of SNe Ia. If we do not include the metallicity effect on SNe Ia, we cannot reproduce the plateau at $[\mathrm{Fe} / \mathrm{H}] \lesssim-1$ (Kobayashi et al. 1998). The scatter is large at $[\mathrm{Fe} / \mathrm{H}]_{\gtrsim}-1$. This may be because the mixing of heavy elements among gas particles has not been included in our chemodynamical model. (d) In the bulge, the $[\mathrm{O} / \mathrm{Fe}]$ plateau continues even at $[\mathrm{Fe} / \mathrm{H}]_{\gtrsim}-1$, but some new stars are also forming at $[\mathrm{Fe} / \mathrm{H}] \sim 0$ with $[\mathrm{O} / \mathrm{Fe}] \sim 0$.

\section{References}

Kobayashi, C., 2004, MNRAS, 347, 740

Kobayashi, C., Springel, V., \& White, S. D. M. 2007, MNRAS, 376, 1465 (K07)

Kobayashi, C., Umeda, H., Nomoto, K., Tominaga, N., \& Ohkubo, T. 2006, ApJ, 653, 1145 